\title{
Users regard operator training as critical for successful POCT applications
}

\author{
Andreas Bietenbeck*1, Katharina Wüst ${ }^{1}$, Peter B. Luppa ${ }^{1}$ \\ 1 Institut für Klinische Chemie und Pathobiochemie; Klinikum rechts der Isar TU München; \\ Germany
}

Corresponding author:

Dr. med. Andreas Bietenbeck

Institut für Klinische Chemie und Pathobiochemie

Klinikum rechts der Isar TU München

Ismaninger Str. 22

81675 München

Germany

andreas.bietenbeck@tum.de

ORCID: http://orcid.org/0000-0002-1228-0770

\section{Abstract}

Point-of-care tests (POCT) measure analytes close to the patients and complementary supplement the test menu of medical laboratories. However, the involvement of many different stakeholders makes it challenging to ensure reliable results. In a survey, we asked experienced POCT users how they control their total POCT process and what factors they consider essential for success. Results were verified in four in-depth interviews.

Overall, 73 German participants from various medical disciplines completed the survey. All but one participant regarded operator training as important but only half of the participants' institutions conducted operator training on a regular basis. Participants often requested elearning, but face-to-face teaching is still preferred. $21 \%$ of participants already used elearning and reported mixed satisfaction. $55 \%$ of the participants never refer to the quality management manual. Instead, $94 \%$ stated that if a POCT error arises a contact person for POCT is always available at their workplace. The majority of participants think that external and in particular internal quality controls are important for POCT. Only a few difficulties for performing quality control such as "temporal expenditure" and "lack of information about the importance of internal quality control" were commonly mentioned. For future developments, participants expect evolution and improvements especially with regard to "measurement quality and reliability". The answers of the experts in the in-depth interviews largely corresponded with the participants of the survey.

The importance of operator training is well established and confirmed in this work. How to conduct this training is less certain but the answers in this survey suggest some form of blended 
learning with e-learning and practical elements. The discrepancy between the high importance that guidelines and other normative documents place on written information and their low practical usage was striking.

\section{Introduction}

Point-of-care testing (POCT) has revolutionized measurements in many medical fields. Glucometers that allow the determination of blood glucose concentration even by the patients themselves have been one of the earliest inventions in this test category $(1,2)$. Similarly, devices that measure blood gases have become indispensable in today's intensive care units (3). Their test menu has been expanded and now often includes co-oximetry, electrolytes and even metabolites such as glucose, lactate, and creatine. All these POCT devices have in common that the measurement is conducted close to the patient. Therefore, the transport of samples is not necessary or reduced to a minimum. Because the operator is usually not a specialist in laboratory medicine, the usage of a POCT device has to be relatively simple. Sample volume can be decreased as pipetting steps are omitted and the sample is directly inserted into the test strip or test cartridge. Samples in POCT are usually measured one by one, in contrast to larger automated analysers in medical laboratories that can process batches of many samples $(4,5)$. With these features, POCT often supplements the test menu of medical laboratories.

Since their introduction, the techniques of POCT devices have improved considerably. With many analytical problems solved, the focus has shifted to the total testing process with extraanalytical issues, as these often determine the medical outcome (6). One important fact is that POCT is a multi-disciplinary process that involves many stakeholders. The physicians or the

nursing team on the wards usually conduct the test, but the specialists in clinical chemistry have to advise the operators and troubleshoot in case of problems. Operator training has to ensure that only qualified personnel employs POCT devices $(7,8)$. In a hospital, the IT department has to connect the POCT devices to the clinical information system to transmit and to store the measured values $(9,10)$. POCT devices usually require large amounts of consumables such as test strips that are vulnerable to conditions like heat or humidity $(11,12)$. The hospital pharmacy or another department has to organize the logistics to reliably provide the ward with these consumables.

Many countries worldwide introduced quality-related regulations for POCT. These include requirements for internal and external quality controls and other tools such as quality management manuals. The introduction of POCT committees has been a major improvement and facilitated the more widespread adoption of $\mathrm{POCT}$, by bringing all these stakeholders together $(13,14)$. These committees often also include a POCT coordinator who works together with POCT representatives in the ward to assist in POCT testing.

While these best practices are widely recognized, it is less well known how POCT is managed in daily routine. To analyse such application-oriented questions, surveys have a long tradition in clinical chemistry. For example, the Q-Probe studies by the College of American Pathologists have used surveys to analyse important quality assurance issues and have influenced the creation of guidelines since 1989 (15-17). In this work, we conducted a survey to discover how 
experienced POCT users control their total point-of-care testing process and which factors they consider essential for success.

\section{Methods}

The POCT survey described here covered several topics. It included basic information such as gender, age, workplace or specific tasks regarding POCT. Moreover, the participants were asked, which POCT methods were used at their workplace. Answer options such as diabetes diagnostics, coagulation diagnostics, diagnosis of cardiovascular diseases, emergency diagnostics (intensive care, emergency medicine), drug screening, neonatology (blood glucose, bilirubin), urine quick tests or microbial rapid tests were given. In addition, performing POCT operations, organisational issues, and quality assurance were important topics. In another part of the survey, the questions dealt with the training of POCT operators and sources of error for POCT. Furthermore, participants also had the opportunity to share their thoughts on the future use of POCT and potential for innovation in this field at the end of the survey.

The survey used open and closed questions. In the open questions, the participants had the opportunity to give answers completely freely. For the closed questions, multiple choice questions with only one option and multiple-choice questions with multiple choices as well as "yes or no" questions were used. In addition, the half-open question was used as an alternative form, which presented both pre-formulated answer options and offered space for free-text answers. Each question was tested for comprehensibility before use. Some questions were only displayed conditionally upon a previous answer (e.g. the participant is only asked to specify training contents if he has completed an operator training). In total, we asked 115 different questions.

The survey was conducted using LimeSurvey version 2.62.2+170203, an online survey tool. The first round took place from 13 to 15 March 2017 during a scientific conference on POCT (18). Here, the questionnaire was divided into four sub-surveys. In the first sub-survey, the main topics were "persons involved in POCT processes", "connection of POCT devices to the IT system" and "organization of POCT including quality management". In the second sub-survey, the main topics were "operator training and e-learning" as well as "hygiene aspects". In the third sub-survey, the main topics were "pre-analytics" and "analytics", whereas in the fourth sub-survey participants were asked about "post-analytics" and the impact of POCT on different occupational groups. In order to shorten the time necessary for completing the survey, each participant was randomly assigned to only one of these sub-surveys. Furthermore, we asked all participants about "general information and demographic data", "methodology and analytical methods" as well as "outlook and future of POCT". The second round of the surveys took place in the period from April to November 2017 in hospitals and POCT laboratories in the Munich area. These participants answered the complete questionnaire and were paid a small expense allowance.

Additionally, four further in-depth telephone interviews with POCT experts were conducted to verify results. In 19 questions, the interviews covered the previous experiences of the experts regarding POCT, which POCT tests they consider most relevant in daily work life, the role of the POCT coordinator, the importance of quality control, and the use of written 
information such as the quality management manual as well as the user's knowledge in terms of POCT guidelines. In addition, the interview examined the utility of operator training and whether e-learning plays a role therein. Possible pre-analytical, analytical, and post-analytical sources of error and ways of avoidance have also been discussed. Finally, the interviewees could state their expectations considering the future development of POCT.

\section{Results}

\section{Participants from various medical specialities}

Overall, 73 participants completed the survey (Table 1). Of these, 54 surveys were conducted 13 to 15 March 2017. The majority of participants worked in large hospitals with more than 800 beds. Participants included physicians, members of the nursing team and laboratory technicians. Other participants with various professions such as assistant, researcher, or chemist were subsumed in an "others" category. 29 participants worked in the central laboratory of a hospital, 10 in POCT sites and 10 in emergency wards.

Table 1. Characteristics of participants:

\begin{tabular}{|c|c|c|}
\hline & $+>$ & Number of participants \\
\hline \multirow[t]{2}{*}{ survey round } & round 1 & 54 \\
\hline & $>1-1$ & 19 \\
\hline \multirow[t]{3}{*}{ age } & $18-35$ years & 22 \\
\hline & $35-60$ years & 44 \\
\hline & $>60$ years & 7 \\
\hline \multirow[t]{2}{*}{$\operatorname{sex}$} & Male & 31 \\
\hline & Female & 42 \\
\hline \multirow[t]{4}{*}{ profession } & Physician & 19 \\
\hline & Nursing team & 12 \\
\hline & Laboratory technologist & 9 \\
\hline & Other & 33 \\
\hline \multirow[t]{6}{*}{ institution } & Central laboratory of a hospital & 29 \\
\hline & nursing home & 2 \\
\hline & private medical practice & 1 \\
\hline & emergency department & 10 \\
\hline & POCT lab & 10 \\
\hline & Hospital ward & 3 \\
\hline-2 & Others & 18 \\
\hline \multirow[t]{8}{*}{ number of beds } & No beds/ only ambulatory care & 26 \\
\hline & 50 - 99 beds & 1 \\
\hline & 100 - 149 beds & 2 \\
\hline & 200 - 299 beds & 4 \\
\hline & 300 - 399 beds & 1 \\
\hline & 500 - 599 beds & 5 \\
\hline & 600 - 799 beds & 1 \\
\hline & 800 and more beds & 33 \\
\hline
\end{tabular}


$67 \%$ ( 8 out of 12) of the members of the nursing team, 44\% (9 out of 9 ) of the laboratory technicians, and $33 \%$ (11 out of 33) of "other" professions carried out at least one POCT test per month. By contrast, only one participant of the group of physicians regularly used POCT himself. The mean numbers of tests in per occupation were 3537.50 (nursing team), 3537.50 (laboratory technicians), and 682.73 (others). In total, $40 \%(20,726)$ of all monthly POCT were tests of blood glucose concentrations, $30 \%(15,450)$ were blood gas analyses, and $18 \%(9,362)$ were urine analyses.

During the March 2017 round, participants answered 30.7 questions on average. The remaining participants answered 76.8 questions on average.

The participants of the in-depth telephone interview included two technical assistants, a sports scientist and a paediatrician with experience in intensive care and emergency medicine. Every interview lasted about 45 minutes.

\section{Operator training is essential for successful POCT}

51 participants provided a free-text response to our question regarding factors for the success of POCT. Of these, $22 \%$ mentioned operator training as an important factor for successful POCT operation. We also asked explicitly, whether training improves the quality of POCT measurements and every participant who answered (31) agreed.

However, $27 \%$ (9 out of 33) of the participants stated that it is not guaranteed at their workplace that only instructed operators perform POCT tests. Half of the participants (51\%, 18 out of 35) answered that their POCT tests require identification of the operator e.g. using a chip card to unlock the measurement. $14 \%$ (5 out of 35) claimed that their POCT device has no built-in operator identification. All of these participants worked in an "other" profession.

For $91 \%$ (30 out of 33) of the participants, an introductory training was given before routine use of POCT. Regular POCT training at the workplace was conducted in $52 \%$ (17 out of 33). For this question, the occupations of the participants were distributed relatively evenly. Training is repeated every year ( $23 \%$, 4 out of 17 ), every six months ( $23 \%, 4$ out of 17 ) or when necessary (35\%, 6 out of 17) for most participants. For $71 \%$ (12 out of 17) of the participants, training is mandatory. The availability of regular training is almost equally common whether a POCT coordinator or other POCT representative is available.

Past training covered a broad range of topics (Fig 1). "Conduction of quality controls" was the most taught topic ( $82 \%, 14$ out of 17), but several other topics occurred in over $70 \%$. Practical training was done in $65 \%$ (11 out of 17 ). 


\section{topics of operator training}

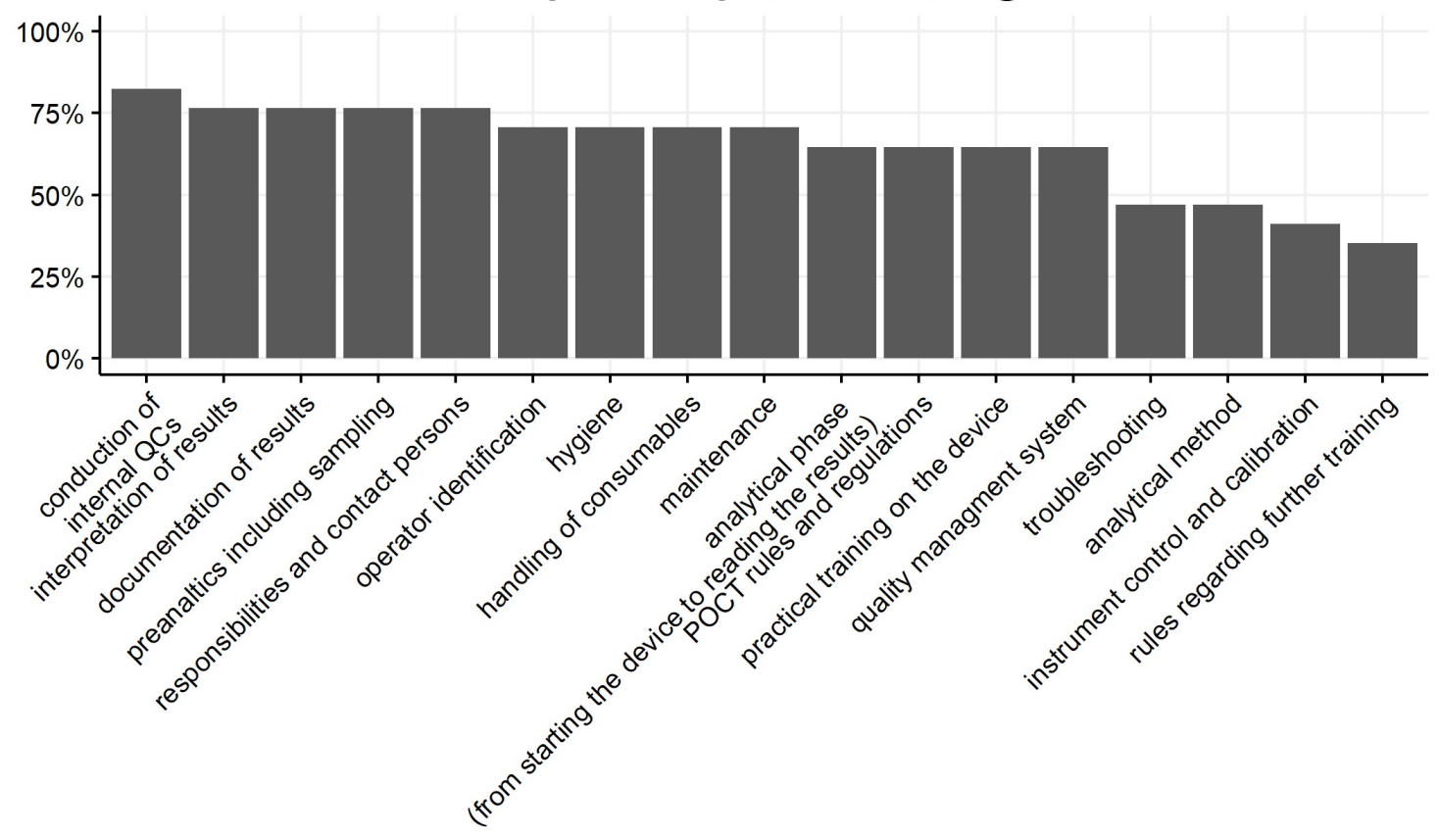

Fig 1. Topics of POCT operator training. POCT operator training covered a broad range of topics. "Conduction of quality controls" was the most taught topic.

Regarding the importance of POCT workplace training, in-depth interviews with experts were consistent with the results of the survey. All rated training as highly relevant to maintaining good quality outcomes. Two of the experts emphasized that training should take place at fixed intervals, independent of new employees or new equipment, as there are always personnel changes and regular appointments can reveal previous deficits. The other experts, on the other hand, stated that a one-time introduction is often sufficient for simple tests and that training should be carried out after major changes only. Overall, however, training courses were declared decisive for the beneficial application of POCT.

\section{E-Learning can supplement face-to-face learning}

$70 \%$ (23 out of 33) of the participants want additional training (Fig 2). 19 participants provided free text answers about desired additional training. Answers were diverse, but 5 (26\%) participants mentioned some form of e-learning. Participants who have no experience with elearning were also explicitly asked if they would like to have this opportunity. $58 \%$ (15 out of 26) agreed. Only 3 out of the 23 participants who want additional training did not want elearning (Fig 2). 


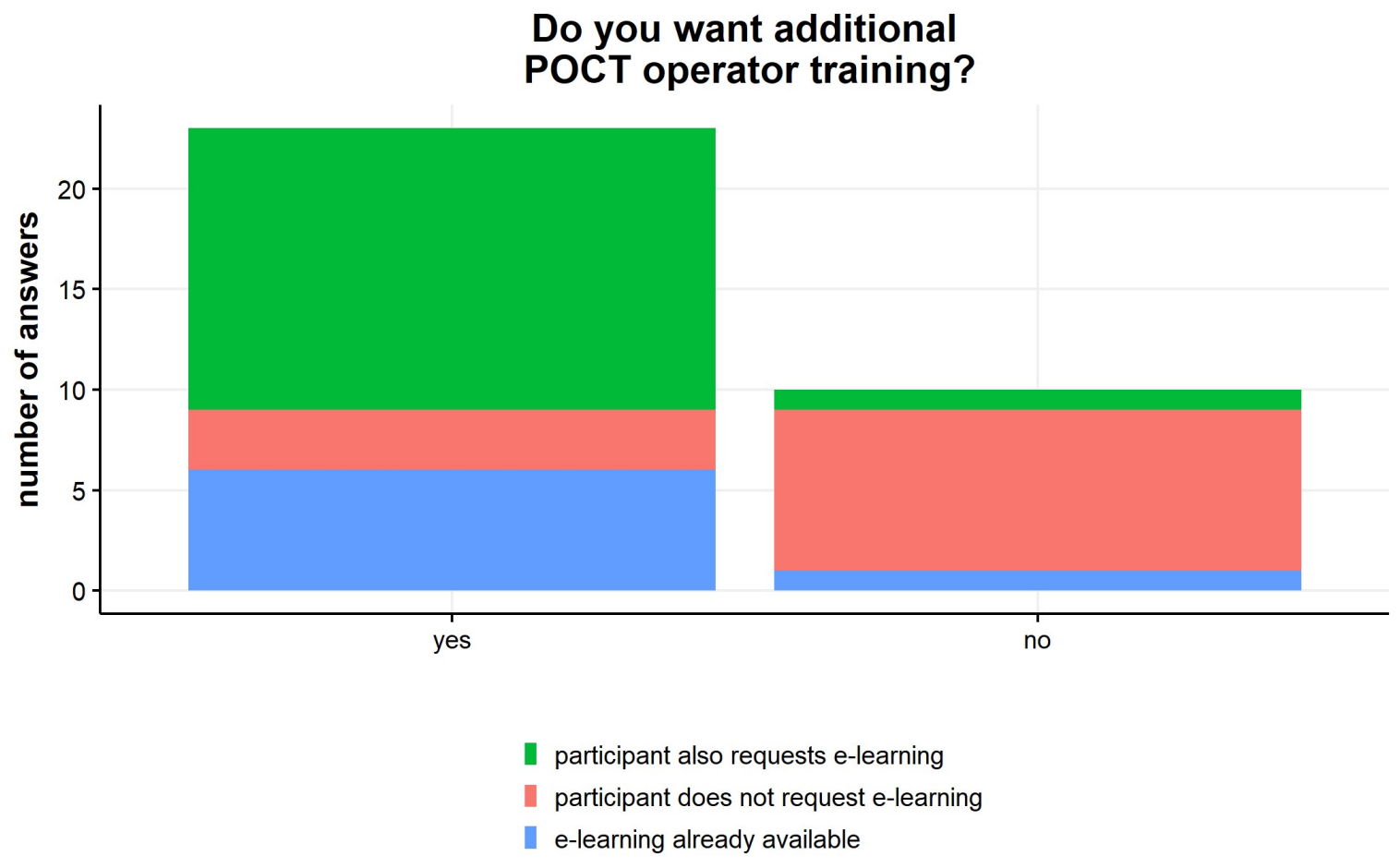

Fig 2. Participants request additional training. $70 \%$ of participants requested additional operator training. Many participants also request e-learning.

$21 \%$ (7 out of 33) of the participants already used e-learning. Satisfaction was mixed with 3 participants being "satisfied", 3 participants being "relatively satisfied" and 1 participant being "rather unsatisfied". When the participants had to choose between e-learning and training with an instructor, $88 \%$ (29 out of 33) preferred the training with an instructor.

In terms of e-learning, the experts' opinions determined in the in-depth interviews differed in part. While one expert praised the great success of introducing such a system in her workplace and highlighted the strong positive response from the staff, another expert criticized e-learning as requiring a high level of self-motivation. It was also mentioned that the use of e-learning depends very much on the workplace conditions. If the employer frees employees to use elearning and integrates it into working hours, these opportunities increase the motivation to carry out e-learning. The experts mentioned that often there is no time to carry out e-learning during daily working routine. The reasons given included unforeseeable emergencies and a shortage of staff. Nonetheless, the experts highlighted the great advantage of e-learning across the workplace, as well as its applicability at any day or night time. For example, this also allows employees in night shifts to retrain without having to attend classroom training during the day.

\section{Written information is less sought after than POCT coordinator}

We assessed how participants sought information. Only 29 \% (9 out of 31) of the participants answered that they consult the quality management manual at least occasionally if questions regarding POCT arise (Fig 3). The majority of participants (55 \%, 17 out of 31) stated that they 
never refer to the quality management manual or that such a manual does not exist in their institution. All participants who do not have a quality management manual available worked in "other" professions (8 out of 31). Remarkable, seven of the nine participants who answered that they never consult a quality management manual were members of the nursing team.

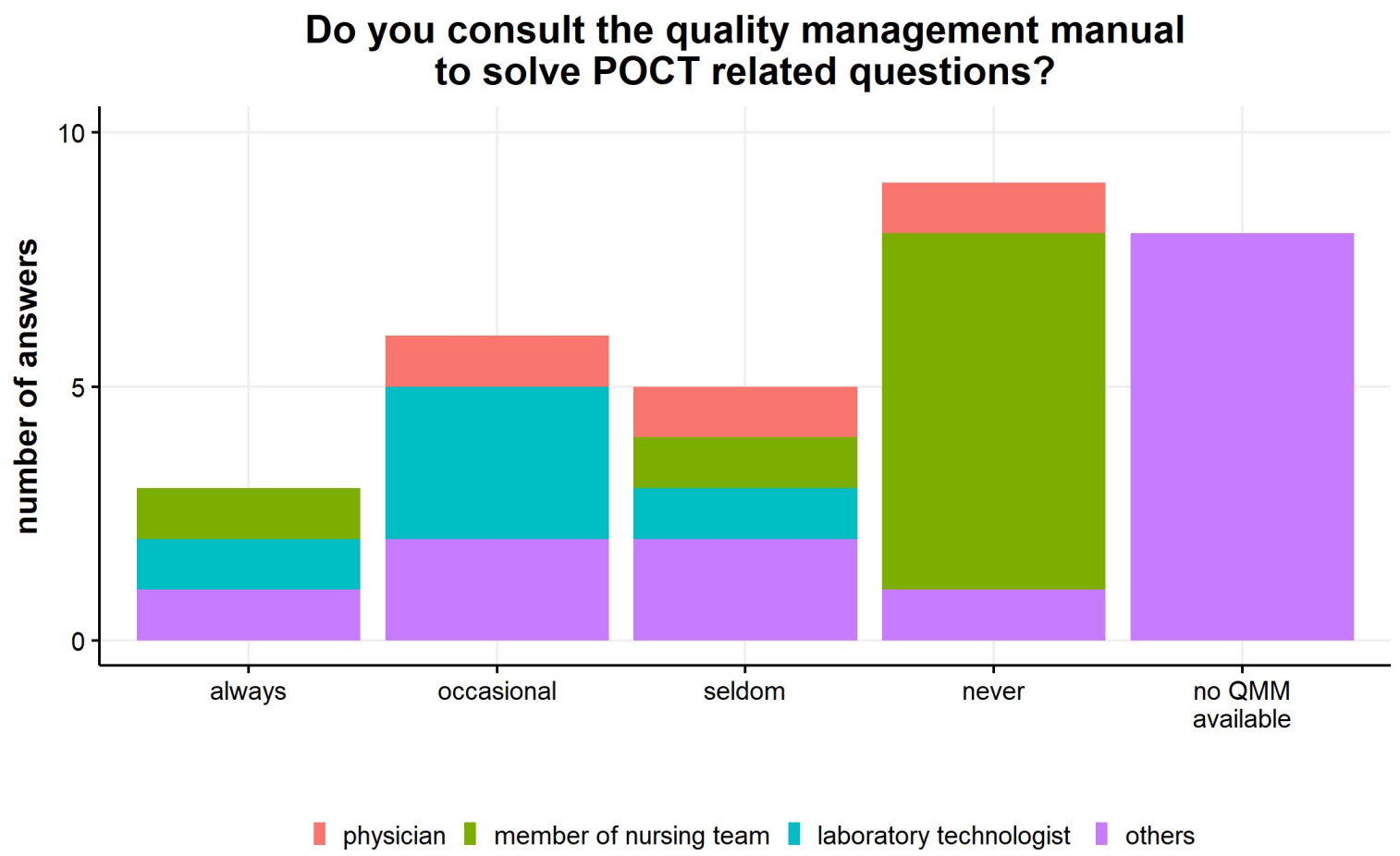

Fig 3. The relevance of the quality management manual for POCT related questions. Especially members of the nursing team never consult the quality management manual.

We also asked participants whether a contact person is always available in case of errors. Only 2 participants (out of 31) negated. Another $77 \%$ (24 out of 31) confirmed that there is a POCT coordinator or a POCT representative at their workplace.

Regarding the relevance of written information, e.g. in form of quality management manuals, the experts in the in-depth interviews also came to the same conclusion as the participants in the survey. They emphasized that, for example, quality management manuals were available at every workplace, but in reality, were hardly ever used. On the other hand, it was stressed that a contact person is very well accepted and appreciated by the POCT operators, as they can be consulted easily and quickly in case of problems. The experts mentioned close communication as a key point.

External and especially internal quality control are deemed important

$45 \%$ (14 out of 29) of the participants regarded internal quality control as "extremely important", another $45 \%$ believed that internal quality controls are "very important" (Fig 4). The remaining $10 \%$ (3 out of 29) saw internal quality control measurements as "relatively important". 
How important is QC for you?

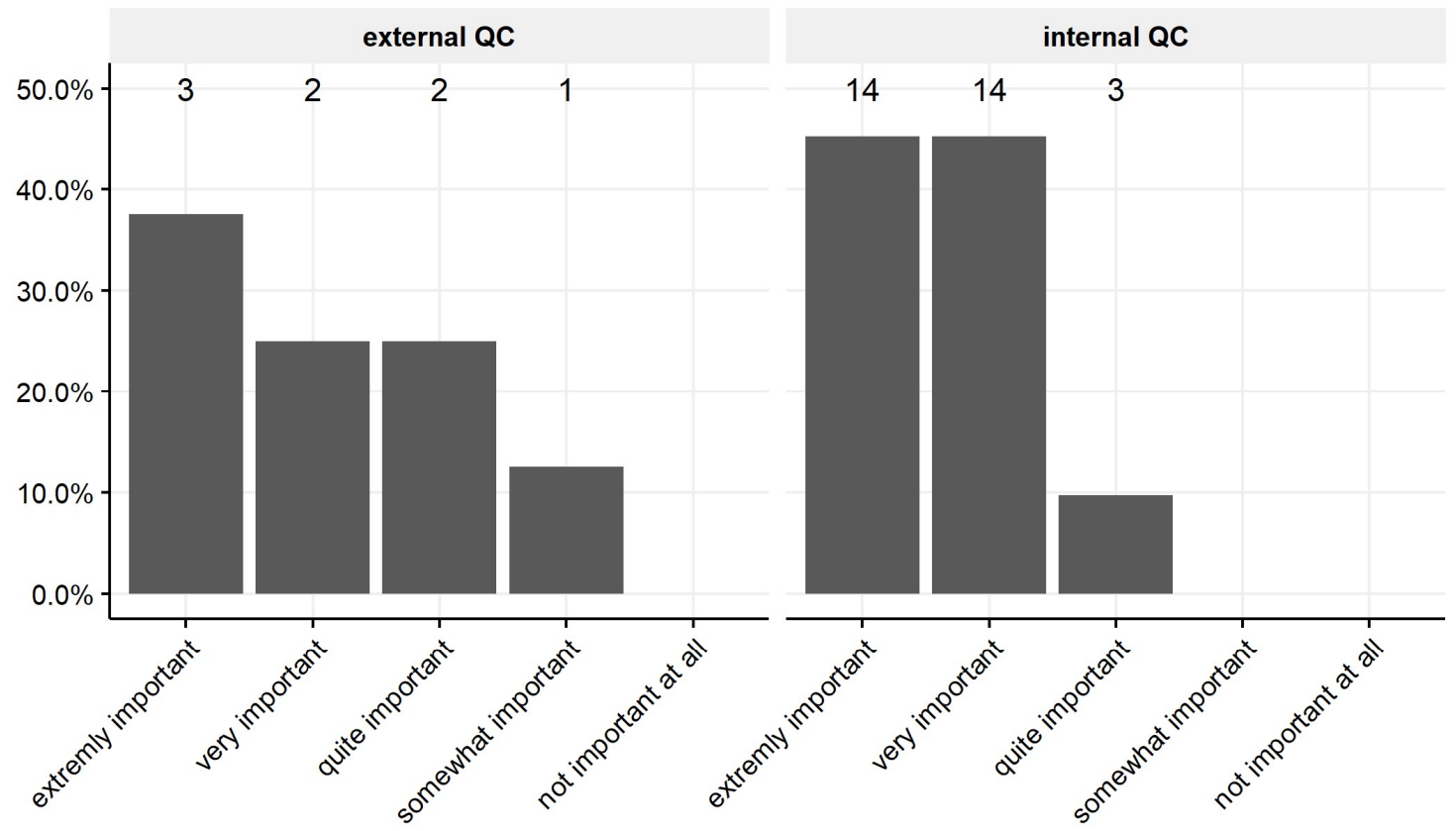

Fig 4. The importance of external and internal quality control. Both external and internal quality controls are held in high regard by the participants of our survey.

Only 8 (out of $31,26 \%$ ) participants stated that they take part in external quality assessment schemes for their POCT measurement. Those, who negated external quality assessment participation, included 12 members of "other" professions but also 9 members of the nursing team and 1 physician. Importance of external quality control was gauged as "extremely important" by $38 \%$ (3 out of 8), as "very important" by $25 \%$ ( 2 out of 8 ), and as "relatively important" by $25 \%$ ( 2 out of 8 ). One participant regarded external quality control as "somewhat important".

We asked about difficulties concerning internal and external quality control. Response options regarding internal quality control included "temporal expenditure", "financial expenditure", "lack of operator competence", "staff shortage" or "lack of information about the importance of internal quality control. "Sample volume", "shipping", "commutability", "evaluation", "interpretation of the evaluation" or "transmitting the results" constituted possible answers respecting external quality control. For both types, we also allowed a free text answer. 31 participants answered the question regarding internal and 8 participants answered the question regarding external quality control. Out of all possible answers, only "temporal expenditure" (58 \%, 18 out of 31) and "lack of information about the importance of internal quality control" (55 \%, 17 out of 31 ) were selected as a difficulty for internal quality control by over $50 \%$ of the participant. No answer reached this ratio of agreements for external quality control.

Internal quality controls are also considered highly significant by all experts of the in-depth interviews. They stated that they are important in order to obtain reliable values and to verify 
that the results of the measurements are correct - preferably in the form of daily quality controls. Regarding problems that may occur during the implementation of internal quality controls, the experts mentioned the lack of acceptance by the some POCT operator, e.g. from the nursing team. This matches the results of the survey.

Participants expect an evolution not a revolution with improvements in POCT quality and reliability

In our last question, we asked participants about their expectations concerning further POCT developments. We received 40 free text answers. We identified the seven recurrent aspects "easier handling", "miniaturization", "cost reduction", "measurement quality and reliability", "turn-around-time", "connectivity" and "parameter spectrum" and clustered the answers accordingly (Fig 5). All aspects were mentioned at least 3 times. 19 participants mentioned "Measurement quality and reliability". Other aspects were mentioned less frequent. One physician mentioned all aspects and members of the nursing team only mentioned "cost reduction", "measurement quality and reliability", and "turn-around-time".

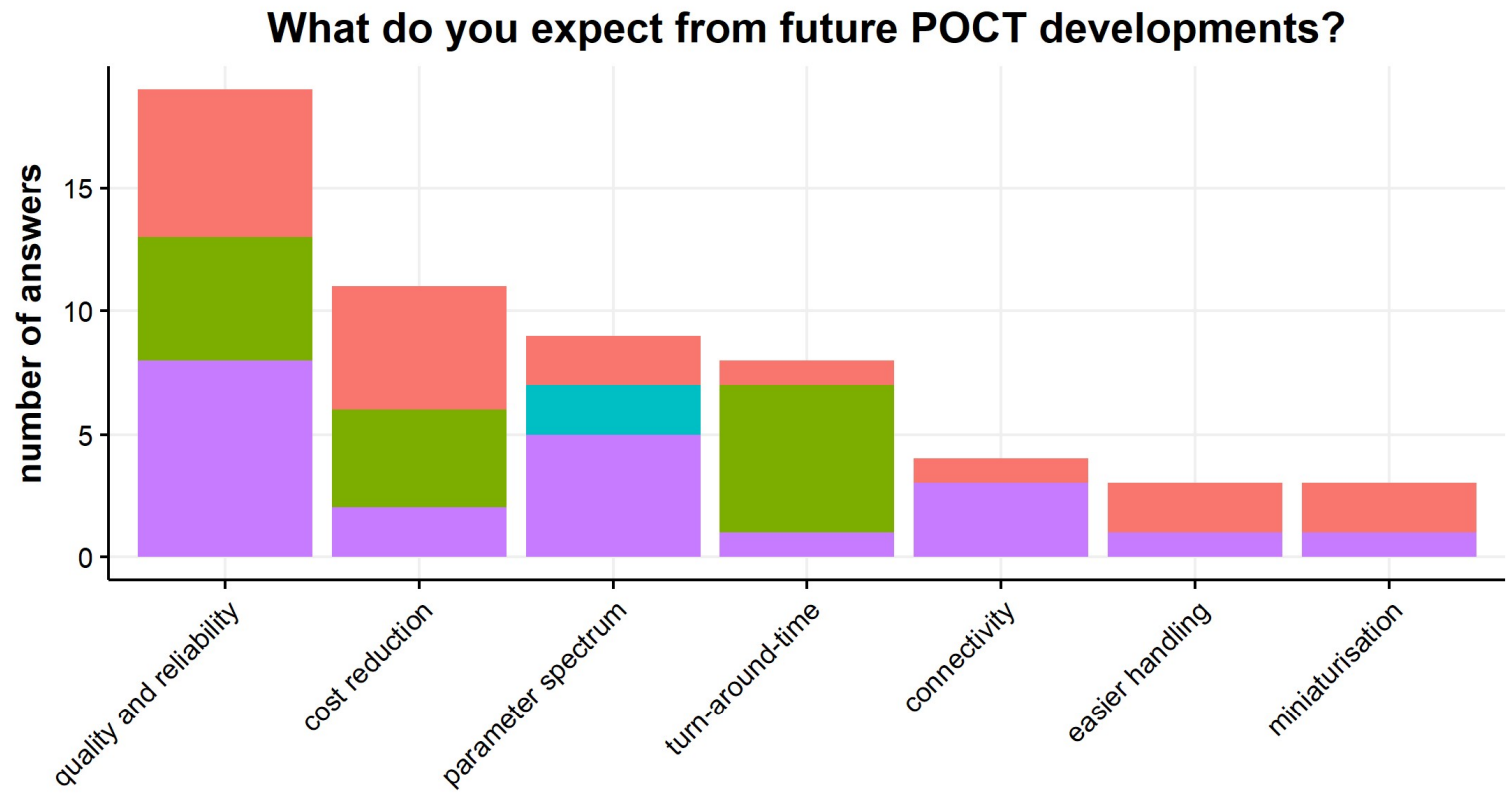

| physician member of nursing team | laboratory technologist | others

Fig 5. Expectations concerning further POCT developments. 40 free text answers were clustered into recurrent aspects.

Referring to future developments, the experts of the in-depth interviews commented on various points that were also mentioned by the participants of the survey. These included the availability of tests with good sensitivity and specificity at reasonable test prices. In addition, tests such as CRP, procalcitonin, or cardiac troponin rapid test for the emergency service were 
requested. The production of so-called customized panels, which enable the simultaneous measurement of an individually compiled constellation of parameters, was also demanded.

\section{Discussion}

For this POCT application study, we conducted an exploratory survey with a broad range of questions. For most questions, we did not collect enough answers to draw conclusions that can be generalized for all POCT users. However, we were able to recruit expert users with very diverse backgrounds reflecting the diverse spectrum of professionals dealing with POCT. The high percentage of free text answers illustrated our participants' high motivation.

Most of our participants agreed that operator training is vital for successful POCT operation. This opinion is well supported by research and has been incorporated into various guidelines (7, 19-24). Uptake into clinical practice, however, seems patchy. While most participants received some form of initial instructions, repeated training was only provided for half of the participants. More than one-fourth of participants were not able to guarantee that only instructed operators perform POCT tests. Better devices, e.g. with the ability to lock out operator with insufficient training seem mandatory to enforce adherence to guidelines (25).

E-learning seems to be a convincing solution to educate a large number of POCT operators. Indeed, the majority of participants who calls for more training also calls for e-learning. The participants who already use e-learning, however, have had mixed experiences. When our participants had to choose between e-learning and instructor based training, almost all preferred training with an instructor. Therefore, it seems best to blend both e-learning and instructor based training into a common concept $(26,27)$.

When asked where our participants sought help in case of POCT problems, contact persons such as POCT representatives or POCT coordinators ranked much higher than written information. Most guidelines and legal documents place their emphasis on written information and neglect other organisation structures such as the POCT coordinator. In clinical practice, there is often no time to search for information in lengthy text documents. In order to be usable, these documents need to be displayed in a more accessible way. Guidelines and legal documents prescribe detailed specifications for written information for POCT. These guidelines should take into account other information sources besides written documents even if these sources are harder to control and verify.

When asked about their expectations regarding future POCT systems our participants expected an evolution with further improvements in the quality and the reliability of POCT devices. They did not share the enthusiasm of many researchers that expect a test menu expansion with new POCT applications such as for personalised medicine (28). This hesitation seems understandable given the difficulties that POCT still faces in daily routine. In order to translate new technologies into clinical practice, these mostly organisational obstacles have to be solved.

\section{Acknowledgments}

This work was supported by the German Research Foundation (DFG) and the Technical University of Munich within the funding programme Open Access Publishing. 


\section{References}

1. Clarke SF, Foster JR. A history of blood glucose meters and their role in self-monitoring of diabetes mellitus. Br J Biomed Sci. 2012;69(2):83-93.

2. Wahl HG, Koschinsky T. Diabetes diagnostics including analytical methods for glucose monitoring. Point-of-Care Testing: Springer; 2018. p. 103-20.

3. Bloom BM, Grundlingh J, Bestwick JP, Harris TJEJoEM. The role of venous blood gas in the emergency department: a systematic review and meta-analysis. 2014;21(2):81-8.

4. Luppa PB, Bietenbeck A, Beaudoin C, Giannetti A. Clinically relevant analytical techniques, organizational concepts for application and future perspectives of point-of-care testing. Biotechnol Adv. 2016;34(3):139-60.

5. St John A, Price CP. Existing and Emerging Technologies for Point-of-Care Testing. The Clinical biochemist Reviews. 2014;35(3):155-67.

6. Hawkins R. Managing the Pre- and Post-analytical Phases of the Total Testing Process. Ann Lab Med. 2012;32(1):5-16.

7. Barabas N, Bietenbeck A. Application guide: training of professional users of devices for nearpatient testing. J Lab Med. 2017;41(5):215.

8. Wiencek J, Nichols J. Issues in the practical implementation of POCT: overcoming challenges. Expert Rev Mol Diagn. 2016;16(4):415-22.

9. Kim JY, Lewandrowski K. Point-of-Care Testing Informatics. Clinics in Laboratory Medicine. 2009;29(3):449-61.

10. Kent Lewandrowski, Kimberly Gregory, Donna Macmillan. Assuring Quality in Point-of-Care Testing: Evolution of Technologies, Informatics, and Program Management. 2011;135(11):1405-14.

11. Tonyushkina K, Nichols JHJJods, technology. Glucose meters: a review of technical challenges to obtaining accurate results. 2009;3(4):971-80.

12. Louie Richard F, Ferguson William J, Curtis Corbin M, Vy John H, Kost Gerald J. Vulnerability of point-of-care test reagents and instruments to environmental stresses: implications for health professionals and developers. Clin Chem Lab Med2014. p. 325.

13. Gässler N, Luppa PB, Bietenbeck A, Petersmann A, Pröbstl A, Romann D, et al. Implementation of POCT. Point-of-Care Testing: Springer; 2018. p. 303-12.

14. Jacobs E, Hinson KA, Tolnai J, Simson EJCca. Implementation, management and continuous quality improvement of point-of-care testing in an academic health care setting. 2001;307(1-2):49-59. 15. Schifman RB, Howanitz PJ, Souers RJ. Point-of-Care Glucose Critical Values: A Q-Probes Study Involving 50 Health Care Facilities and 2349 Critical Results. Arch Pathol Lab Med. 2016;140(2):11924.

16. Dyhdalo KS, Howanitz PJ, Wilkinson DS, Souers RJ, Jones BA. Documentation of Quality Control and Operator Training at Point-of-Care Testing: A College of American Pathologists Q-Probes Study of 106 Institutions. 2014;138(11):1444-8.

17. Nakhleh RE, Souers RJ, Bashleben CP, Talbert ML, Karcher DS, Meier FA, et al. Fifteen Years' Experience of a College of American Pathologists Program for Continuous Monitoring and Improvement. 2014;138(9):1150-5.

18. Bietenbeck A, Schmalenberg $M$, Luppa Peter B. Kongressbericht: 3. Münchner POCTSymposium, 13. - 15. März 2017, Klinikum rechts der Isar der TU München. LaboratoriumsMedizin2017. p. 205.

19. New Zealand Best Practice Guidelines For Point-of-Care Testing, (2014).

20. Medical laboratories: requirements for quality and competence, (2012).

21. German Medical Association. Revision of the "Guideline of the German Medical Association on Quality Assurance in Medical Laboratory Examinations-RiliBAEK". J Lab Med. 2015;39(1):26-69. 
22. Dolscheid-Pommerich RC, Dolscheid S, Grigutsch D, Stoffel-Wagner B, Graeff IJPo. Comparability of point-of-care versus central laboratory hemoglobin determination in emergency patients at a supra-maximal care hospital. 2016;11(11):e0166521.

23. Liikanen E, Lehto L. Training of nurses in point-of-care testing: a systematic review of the literature. 2013;22(15-16):2244-52.

24. Taylor BM, DeJear L, Teehee M. The role of education and point of care use in appropriate surface disinfection compliance. 2013;41(6):S130.

25. Fadlalla N. POCT Testing and Importance of Operator Lock-out: Dominican University of California; 2018.

26. Edwards G, Kitzmiller RR, Breckenridge-Sproat S. Innovative Health Information Technology Training: Exploring Blended Learning. 2012;30(2):104-9.

27. Lehto LA, Bloigu A, Liikanen E, Ruokonen AJPoC. Interactive 2-step strategy for training nurses: a practical tool for achieving better-quality point-of-care glucose testing in hospital and primary health care unit. 2014;13(2):41-7.

28. Ahmed MU, Saaem I, Wu PC, Brown ASJCrib. Personalized diagnostics and biosensors: a review of the biology and technology needed for personalized medicine. 2014;34(2):180-96. 